\title{
A New Distance Relaying Algorithm for Series Compensated Transmission Lines Based on Micro-Processor
}

\author{
Yuchao Qiao ${ }^{1, a}$, Zhenyu $\mathrm{Xu}^{1 \text {, a }}$, Jiangwen Meng ${ }^{1, \mathrm{~b}}$ and Shupeng $\mathrm{Li}^{1, \mathrm{~b}}$ \\ ${ }^{1}$ School of Electrical and Electronic Engineering, North China Electric Power university, \\ Beijing,102206, China \\ ahfgdqyc@163.com, b670269734@qq.com
}

Keywords: Distance relaying algorithm, series compensated transmission lines, fault impedance, fault transient process.

\begin{abstract}
A new and simple distance relaying algorithm based on KVL for series compensated transmission lines is discussed in this paper. The fault impedance can be calculated accurately by solving the 3-order fault loop equations consisting of fault phase voltage, current, the differential and integral of current. In theory, it studies the applicability of the scheme in fault transient process, and put forward the corresponding solution. The simulation test shows that the proposed algorithm operates better than the traditional scheme for the series compensated transmission lines.
\end{abstract}

\section{Introduction}

Applying series compensation in power systems can increase power transfer capability, improve transient stability and damp power oscillations [1]. Even the series compensation technology has many advantages, it also brings difficulties to the line protection. The conventional protection will lose its selectivity or direction. The placement site of the series capacitor will affect the protection performance of the series compensated line. In addition, the extreme nonlinear characteristic of metal oxide arrestor (MOV) may cause overreach for the traditional distance relaying scheme [2].

To eliminate the influence of series capacitor and the nonlinear characteristic of MOV, many schemes has developed in the past few years. Firstly, it shortens the reaching setting of the conventional distance protection to avoid the impact of the series capacitor [3]. Secondly, [4, 5] proposed the improvement for directional and pilot protection on series-compensated lines, however, the scheme can't be applied to step distance relay in the practical engineering. And then, [6, 7]used the wavelet transform technique to improve the performance of protection on series compensated transmission lines. However, all these methods need further observation.

The scheme suggested in [8] can calculate the fault impendence correctively without requirement of the parameters of series capacitor device. It utilizes a three order equation group based on KVL to calculate the fault reactance $X$ and fault resistance $R$. Mathematically, the equation group above may be free of solution, or have numerous solutions. Corresponding to the problem of number of solutions for the equation group, the scheme proposed in [8] is invalid if the equation group is free of solution. It analyses the problem above and develops the corresponding solution project in this paper.

\section{Theory Basis}

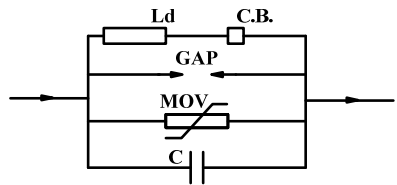

(a) Fixed SC

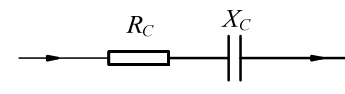

(b) Equivalent circuit

Fig.1. SC/MOV/GAP Equivalent Circuit for Analysis

Fig.1.(a) shows the structure of modern SC/MOV/GAP device. In order to protect SC from overvoltage, MOV and GAP are installed in parallel with SC. The series capacitor SC/MOV can be linearized as the series connection of $R \mathrm{c}$ and $X \mathrm{c}$ when the MOV conducts [9]. Fig.1.(b) presents the 
equivalent circuit of SC/MOV/GAP. Three different operating states incorporate the SC/MOV/GAP different protection functions for different fault conditions. The three operating states are as follows:

1) $\mathrm{SC} / \mathrm{MOV}$ is bypassed with GAP discharging corresponding to $R \mathrm{c}=X \mathrm{c}=0$;

2) SC operates with MOV conducting and without GAP discharging corresponding to $R \mathrm{c} \neq 0, X \mathrm{c}$ $\neq 0$;

3) SC operates without MOV conducting and GAP discharging corresponding to $R \mathrm{c}=0, X \mathrm{c} \neq 0$.

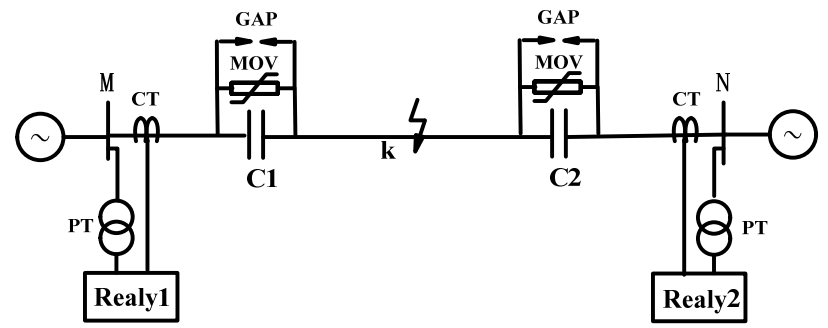

Fig.2. Series compensated transmission line

The test model used is a 500kV series compensated transmission line with $300 \mathrm{~km}$ long as shown in Fig.2.

Firstly, for the three order homogeneous linearity equations, as shown (1) $A x=b$

1) If $r(A)=r(A, b)=3$, the equations above have unique solutions;

2) If $r(A)=r(A, b)<3$, the equations above have numerous solutions;

3) If $r(A)<r(A, b)$, the equations above have no solutions.

Where, $r(\mathrm{~A})$ is rank of matrix $\mathrm{A}$.

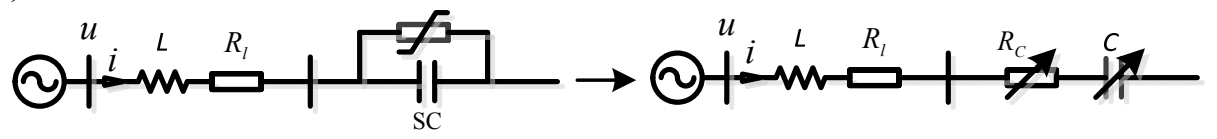

Fig.3. The equivalent circuit of series compensated line during fault period

When a fault occurs on the line, neglecting the distributed capacitance, the phase (take phase A as example) voltage of the bus $\mathrm{M}$ is shown as follow:

$$
u_{A}=u_{k A}+i_{A 1} R_{1}+i_{A 2} R_{2}+i_{A 0} R_{0}+L_{1} \frac{d i_{A 1}}{d t}+L_{2} \frac{d i_{A 2}}{d t}+L_{0} \frac{d i_{A 0}}{d t}+\frac{1}{C_{1}} \int_{t 0}^{t} i_{A 1} d t+\frac{1}{C_{2}} \int_{t 0}^{t} i_{A 2} d t+\frac{1}{C_{0}} \int_{t 0}^{t} i_{A 0} d t
$$

Where, $u_{A}$ is the voltage of phase A at bus $\mathrm{M}, u_{k A}$ is the voltage of phase A at fault point, $i_{1}, i_{2}, i_{0}$ are the positive, negative, zero sequence current at bus $\mathrm{M}, R_{1}, R_{2}, R_{0}, L_{1}, L_{2}, L_{0}$ are positive, negative, zero sequence resistance and reactance of series compensated line, $C_{1}, C_{2}, C_{0}$ are positive, negative, zero sequence series capacitance.

For the high voltage transmission lines, $R_{1}=R_{2}, L_{1}=L_{2}, C_{1}=C_{2}=C_{0}$. (2) can be written as (3)

$$
u_{A}=R\left(i_{A}+3 i_{A 0} k_{r}\right)+L \frac{d\left(i_{A}+3 i_{A 0} k_{x}\right)}{d t}+\frac{1}{C} \int_{t 0}^{t} i_{A} d t+u_{k A}
$$

Where, $k_{r}=\frac{R_{0}-R_{1}}{R_{1}}, k_{x}=\frac{L_{0}-L_{1}}{L_{1}}$ are zero sequence compensation coefficient.

Similarly, for phase B and C

$$
\begin{aligned}
& u_{B}=R\left(i_{B}+3 i_{B 0} k_{r}\right)+L \frac{d\left(i_{B}+3 i_{B 0} k_{x}\right)}{d t}+\frac{1}{C} \int_{t 0}^{t} i_{B} d t+u_{k B} \\
& u_{C}=R\left(i_{C}+3 i_{C 0} k_{r}\right)+L \frac{d\left(i_{C}+3 i_{C 0} k_{x}\right)}{d t}+\frac{1}{C} \int_{t 0}^{t} i_{C} d t+u_{k C}
\end{aligned}
$$

\section{A. Single Phase to Ground Fault}

When AG solid fault occurs on the transmission line, $u_{k A}=0,(3)$ can be written as

$$
u_{A}=R\left(i_{A}+3 i_{A 0} k_{r}\right)+L \frac{d\left(i_{A}+3 i_{A 0} k_{x}\right)}{d t}+\frac{1}{C} \int_{t 0}^{t} i_{A} d t
$$

According to [7], differentiating (6) with respects to $t$, (7) can be obtained 


$$
\frac{d u_{A}}{d t}=R \frac{d\left(i_{A}+3 i_{A 0} k_{r}\right)}{d t}+L \frac{d^{2}\left(i_{A}+3 i_{A 0} k_{x}\right)}{d t^{2}}+\frac{1}{C} i_{A}
$$

A three order equation group consists of arbitrary three discontinuous sampling points satisfying (7), then the fault reactance $X$ and fault resistance $R$ can be obtained. The equation group is as follow

$$
\left[\begin{array}{lll}
{\left[\frac{d\left(i_{A}+3 i_{A 0} k_{r}\right)}{d t}\right]_{t 1}} & {\left[\frac{d^{2}\left(i_{A}+3 i_{A 0} k_{x}\right)}{d t^{2}}\right]_{t 1}} & \left(i_{A}\right)_{t 1} \\
{\left[\frac{d\left(i_{A}+3 i_{A 0} k_{r}\right)}{d t}\right]_{t 2}} & {\left[\frac{d^{2}\left(i_{A}+3 i_{A 0} k_{x}\right)}{d t^{2}}\right]_{t 2}} & \left(i_{A}\right)_{t 2} \\
{\left[\frac{d\left(i_{A}+3 i_{A 0} k_{r}\right)}{d t}\right]_{t 3}} & {\left[\frac{d^{2}\left(i_{A}+3 i_{A 0} k_{x}\right)}{d t^{2}}\right]_{t 3}} & \left(i_{A}\right)_{t 3}
\end{array}\right]\left[\begin{array}{l}
R \\
L \\
\frac{1}{C}
\end{array}\right]=\left[\begin{array}{l}
\left(\frac{d u_{A}}{d t}\right)_{t 1} \\
\left(\frac{d u_{A}}{d t}\right)_{t 2} \\
\left(\frac{d u_{A}}{d t}\right)_{t 3}
\end{array}\right]
$$

\section{B. Interphase Fault}

The study presents $\mathrm{AB}$ interphase solid fault as an example, when $\mathrm{AB}$ interphase solid fault occurs on the transmission line, $u_{k A}=0, u_{k A B}=0$, (3) (4) can be written as

$$
\begin{aligned}
& u_{A}=R\left(i_{A}+3 i_{A 0} k_{r}\right)+L_{1} \frac{d\left(i_{A}+3 i_{A 0} k_{x}\right)}{d t}+\frac{1}{C} \int_{t 0}^{t} i_{A} d t \\
& u_{B}=R\left(i_{B}+3 i_{B 0} k_{r}\right)+L_{1} \frac{d\left(i_{B}+3 i_{B 0} k_{x}\right)}{d t}+\frac{1}{C} \int_{t 0}^{t} i_{B} d t
\end{aligned}
$$

Subtracting the two equations above, (11) can be obtained

$$
u_{A B}=R i_{A B}+L_{1} \frac{d i_{A B}}{d t}+\frac{1}{C} \int_{t 0}^{t} i_{A B} d t
$$

Similarly, A three order equation group consists of arbitrary three discontinuous sampling points satisfy (10), then the fault reactance $X$ and fault resistance $R$ can be obtained. The equation group is as follow

$$
\left[\begin{array}{ccc}
\left(\frac{d i_{A B}}{d t}\right)_{t 1} & \left(\frac{d^{2} i_{A B}}{d t^{2}}\right)_{t 1} & \left(i_{A B}\right)_{t 1} \\
\left(\frac{d i_{A B}}{d t}\right)_{t 2} & \left(\frac{d^{2} i_{A B}}{d t^{2}}\right)_{t 2} & \left(i_{A B}\right)_{t 2} \\
\left(\frac{d i_{A B}}{d t}\right)_{t 3} & \left(\frac{d^{2} i_{A B}}{d t^{2}}\right)_{t 3} & \left(i_{A B}\right)_{t 3}
\end{array}\right]\left[\begin{array}{l}
R \\
L \\
\frac{1}{C}
\end{array}\right]=\left[\begin{array}{l}
\left(\frac{d u_{A B}}{d t}\right)_{t 1} \\
\left(\frac{d u_{A B}}{d t}\right)_{t 2} \\
\left(\frac{d u_{A B}}{d t}\right)_{t 3}
\end{array}\right]
$$

\section{Problem existing}

After the power system reaching steady state when permanent fault occurs on the transmission lines, the phase voltage and current at bus $\mathrm{M}$ are sinusoidal.

For the single phase to ground solid fault, assuming that the fault phase current is $i=I_{m} \sin (\omega \mathrm{t}+\theta)$, zero sequence current is $i_{0}=I_{0} \sin (\omega \mathrm{t}+\beta)$ the fault phase voltage is $u=U_{m} \sin (\omega \mathrm{t}+\alpha)$, as shown in (8), the elements of the equation group are as follows

$$
\begin{aligned}
& \frac{d\left(i_{\varphi}+3 k_{r} i_{0}\right)}{d t}=\omega I_{m} \cos (\omega t+\theta)+3 \omega k_{r} \cos (\omega t+\beta), \frac{d^{2}\left(i_{\varphi}+3 k_{x} i_{0}\right)}{d t^{2}}=-\omega^{2} I_{m} \sin (\omega t+\theta)-3 \omega^{2} k_{x} \sin (\omega t+\beta) \\
& \frac{d u_{\varphi}}{d t}=\omega U_{m} \cos (\omega \mathrm{t}+\alpha), \quad i_{\varphi}=I_{m} \sin (\omega t+\theta)
\end{aligned}
$$

As shown above, the elements of the equation group (8) are linearly independent, so $r(A)=r(A, b)=3$, and the corresponding equation group has unique solutions.

For the interphase solid fault, assuming that the fault interphase current is $i_{\varphi \varphi}=I_{m} \sin (\omega \mathrm{t}+\theta)$, the fault interphase voltage is $u_{\varphi \varphi}=U_{m} \sin (\omega \mathrm{t}+\alpha)$, as shown in (12), the elements of the equation group are as follows

$$
\frac{d u_{\varphi \varphi}}{d t}=\omega U_{m} \cos (\omega \mathrm{t}+\alpha), \quad \frac{d i_{\varphi \varphi}}{d t}=\omega I_{m} \cos (\omega t+\theta), \frac{d^{2} i_{\varphi \varphi}}{d t^{2}}=-\omega^{2} I_{m} \sin (\omega t+\theta), \quad i_{\varphi \varphi}=I_{m} \sin (\omega t+\theta)
$$


As shown above, $\frac{d^{2} i_{\varphi \varphi}}{d t^{2}} / i_{\varphi \varphi}=-\omega^{2}$, the elements of the equation group (8) are linearly dependent, so $\mathrm{r}(\mathrm{A})=2, \mathrm{r}(\mathrm{A}, \mathrm{b})=3$, and the corresponding equation group has no solutions.

Above all, the equation group corresponding single phase to ground fault has unique solutions while the equation group corresponding interphase fault has unique solutions. So the scheme proposed in [7] is no longer applicable for interphase fault if the equation group uses power frequency short voltage and current when the power system reach steady state after the fault occurred. It is necessary to analyze the applicability of the proposed scheme under transient state.

\section{Feasibility analysis of the scheme under transient state}

\section{A. Fault behind the capacitor}

By the power system fault analysis theory, the transient current expression is:

$$
i=I_{m} \sin (\omega \mathrm{t}+\theta-\varphi)+I_{0} \sin \left(\omega_{0} \mathrm{t}+\alpha\right) e^{-\frac{t}{T_{a}}}
$$

Where, $I_{m}$ is the amplitude of steady state component of short current, $\varphi$ is the line impedance angle after fault, $I_{0}$ is the initial value of transient oscillating component, $\omega_{0}$ is angular frequency of damped oscillating components, $T_{a}$ is decay time constant, $T_{a}=2 L / R$.

For the ultra high voltage transmission line, $2 L \square R$. Assuming that $T_{a}=\infty$, (13) can be written as (14)

$$
i=I_{m} \sin (\omega \mathrm{t}+\theta-\varphi)+I_{0} \sin \left(\omega_{0} \mathrm{t}+\alpha\right)
$$

For the elements of (12)

$$
\begin{aligned}
& \frac{d i}{d t}=\omega I_{m} \cos (\omega t+\theta-\varphi)+\omega_{0} I_{0} \cos (\omega t+\alpha), \frac{d^{2} i}{d t^{2}}=-\omega^{2} I_{m} \sin (\omega t+\theta-\varphi)-\omega_{0}{ }^{2} I_{0} \sin (\omega t+\alpha) \\
& i=I_{m} \sin (\omega \mathrm{t}+\theta-\varphi)+I_{0} \sin \left(\omega_{0} \mathrm{t}+\alpha\right)
\end{aligned}
$$

As shown above, the elements of the equation group (8) are linearly independent if $\omega \neq \omega_{0}$, so $r(A)=r(A, b)=3$, and the corresponding three order equation group has unique solutions.

The situation of $\omega=\omega_{0}$ is seldom appear because the system reactance is large while it is necessary to avoid the situation of $\omega=\omega_{0}$ in the design stage. In fact, even $\omega=\omega_{0}$, there is other frequency component in the fault current to guarantee that the equation group have solutions.

\section{B. Fault in front of the capacitor}

The series capacitor is not in the fault loop in this case, the fault current is

$$
i=I_{m} \sin (\omega \mathrm{t}+\theta-\varphi)+I_{0} e^{-\frac{t}{T_{a}}}
$$

For the elements of (12)

$\frac{d i}{d t}=\omega I_{m} \cos (\omega t+\theta-\varphi)-\frac{1}{T_{a}} e^{-\frac{t}{T_{a}}}, \frac{d^{2} i}{d t^{2}}=-\omega^{2} I_{m} \sin (\omega t+\theta-\varphi)+\frac{1}{T_{a}^{2}} e^{-\frac{t}{T_{a}}}, i=I_{m} \sin (\omega \mathrm{t}+\theta-\varphi)+I_{0} e^{-\frac{t}{T_{a}}}$

As shown above, the elements of the equation group (8) are linearly independent if $I_{0} \neq 0$, so $r(A)=r(A, b)=3$, and the corresponding three order equation group has unique solutions.

However, the corresponding three order equation group has no solutions if $I_{0}=0$ when the capacitor is not in the fault loop. In this case, monitoring the value of $\Delta(\mathrm{A})$, determinant of matrix $A$,in the first few cycles after fault occurring could recognize that the fault is behind or in front of the capacitor. If $\Delta(\mathrm{A})=0$, the fault is in front of the capacitor, otherwise, the fault is behind the capacitor. Once the fault is in front of the capacitor, the fault impedance can be solved by the two order equation group. For the fault behind capacitor, the transient low frequency component always exists in the fault current no matter how much the initial phase angle is. 


\section{Simulation tests}

In order to evaluate the performance of the novel algorithm, a simulation model of a $500 \mathrm{kV}$, $300 \mathrm{~km}$ series compensated transmission lines is shown in Fig.2. The parameters are presented in the Appendix. PSCAD and MATLAB are utilized for the power system simulation and algorithm calculation, respectively. A two order Chebyshev- II filter is utilized. The least square method is employed to solve (8) (12). Power angle of source $\mathrm{N}$ lags behind source $\mathrm{M}$ by $30^{\circ}$ (heavy load) to $0^{\circ}$ (no load). Distance protection Zone-I is set to protect $85 \%(255 \mathrm{~km})$ of the transmission line. Faults are initiated at $0.4 \mathrm{~s}$. Power frequency is $50 \mathrm{~Hz}$. The sampling rate of 50 samples per cycle (for $50 \mathrm{~Hz}$ system) is used. To compare with the proposed algorithm, the traditional distance relay algorithm for single phase to ground fault and phase to phase fault as shown as (16) and (17) are employed.
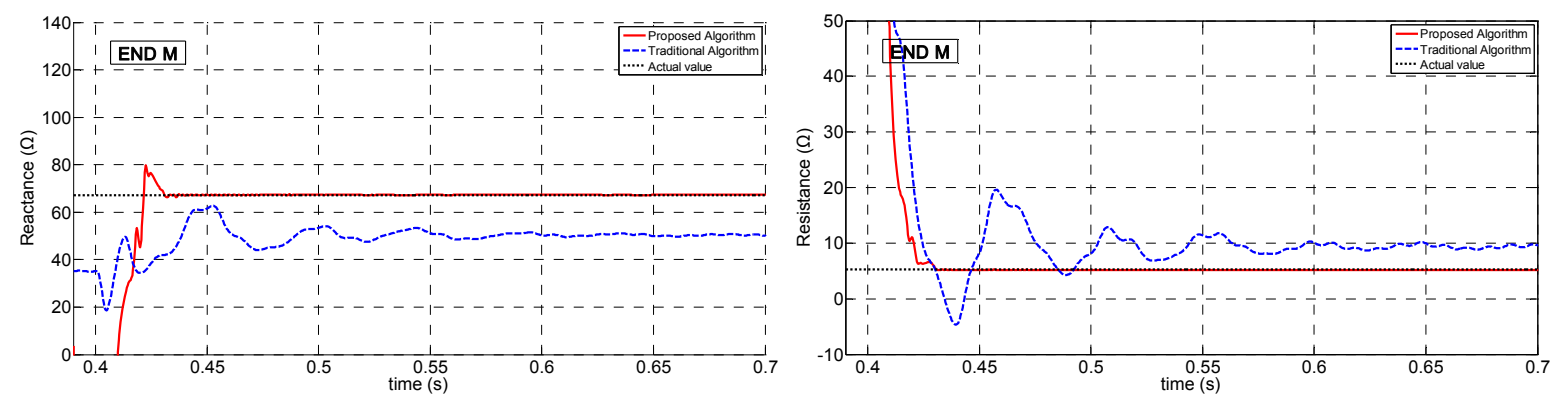

(a) Fault impedance loci measured at end $\mathrm{M}$
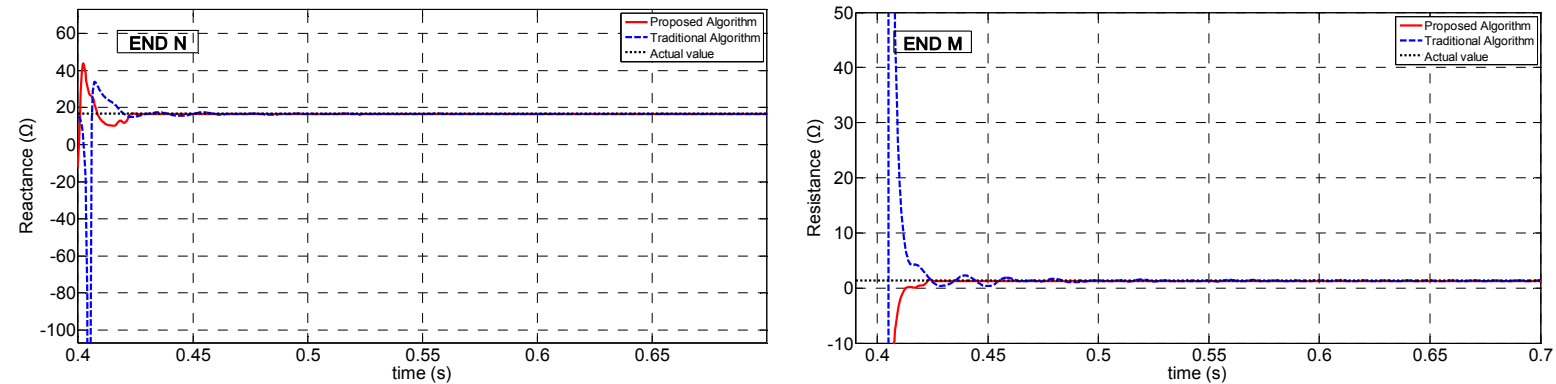

(b) Fault impedance loci measured at end $\mathrm{N}$

Fig.4. Estimated fault impedance under AG solid fault at $240 \mathrm{~km}$ from bus M.
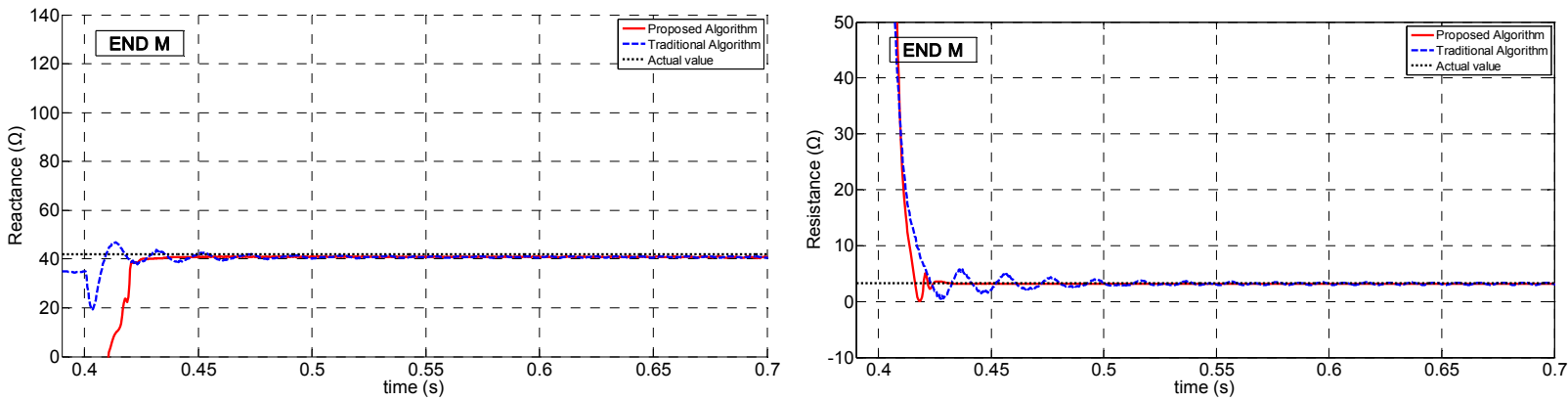

(a) Fault impedance loci measured at end $\mathrm{M}$
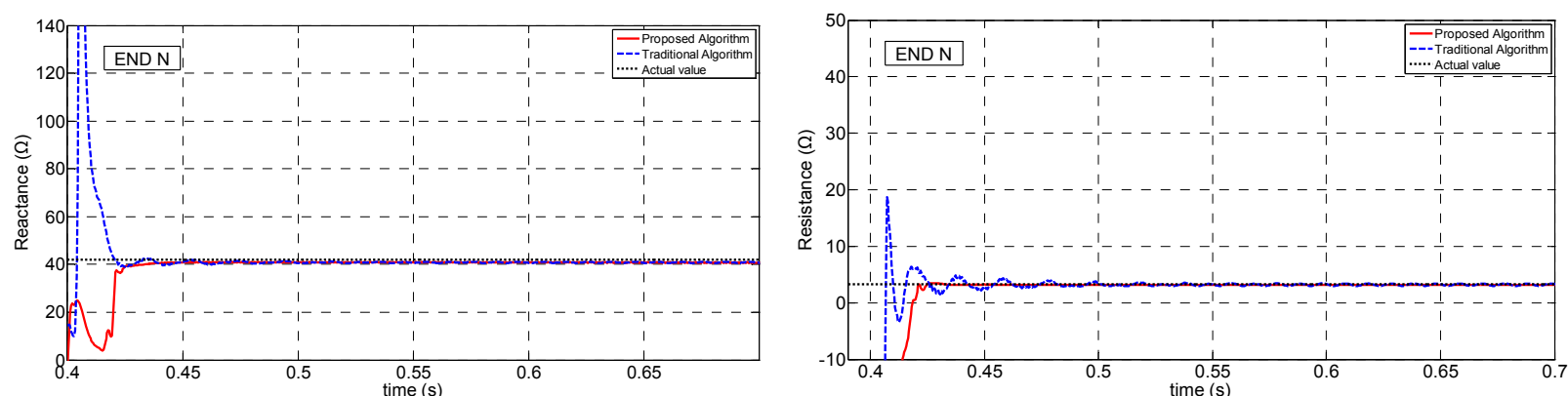

(b) Fault impedance loci measured at end $\mathrm{N}$

Fig.5. Estimated fault impedance under $\mathrm{AB}$ solid phase to phase fault at $150 \mathrm{~km}$ from bus M. $Z_{\varphi}=U_{\varphi} /\left(I_{\varphi}+3 K I_{0}\right)$ 


$$
Z_{\varphi \varphi}=U_{\varphi \varphi} / I_{\varphi \varphi}
$$

Where $K=\left(z_{0}-z_{1}\right) / 3 z_{1}, \varphi$ can be any phase of A, B, or C. $z_{0}, z_{1}$ represent zero-, positive-sequence impedance per unit length, $\varphi \varphi$ can be any phases of $\mathrm{AB}, \mathrm{BC}$, or $\mathrm{CA}$.

Fig.4. shows the comparison results between the proposed algorithm and the conventional algorithm when a AG solid fault occurs at $240 \mathrm{~km}$ away from end $\mathrm{M}$, which is inside the both protected range of relay 1 and relay 2. As shown in Fig.4.(a) and Fig.4.(b), the calculated fault impedance in end $\mathrm{M}$ and $\mathrm{N}$ with the conventional algorithm (16) shows considerable oscillation and inside the first-zone due to the series capacitors being in fault loop. However, the proposed algorithm can calculate the fault impedance accurately, and it will reach stable in about $20 \mathrm{~ms}$ for the proposed algorithm in both ends.

For the phase to phase fault, as the fault phase current is larger than the fault phase current in the case of single phase fault when the fault occurs in the same point on the lines. Generally, for the phase to phase fault, MOV may be conducted, even GAP would be triggered. Fig.5. shows the comparison results between the proposed algorithm and the conventional algorithm (17) when a AB solid phase to phase fault occurs at $150 \mathrm{~km}$ away from end $\mathrm{M}$, which is inside the both protected range of relay 1 and relay 2. As shown in Fig.5.(a) and Fig.5.(b), the calculated fault impedance at relay 1 with the conventional algorithm and proposed algorithm are almost same because the series capacitor in fault loop is bypassed by GAP. However, the calculated fault impedance with proposed algorithm will reach stable in about $20 \mathrm{~ms}$ in both ends.

\section{Conclusion}

By the analysis above, it is reasonable that using superposition of transient and steady state component after fault to calculate the impedance for the proposed scheme. For the ultra high voltage transmission line, the decay time constant is large as the value of $L / R$ is large. So the transient component in the fault current is abundant in the first few cycles after the fault occurred. And the proposed scheme is reliable for single phase to ground fault and interphase fault on the series compensated transmission lines.

\section{References}

[1] Seymour RS, Starr EC . Economic aspect of series capacitors in high voltage transmission[J]. AIEE Trans, PAS, 1951, 5(8): 1660-1670.

[2] F. Ghassemi, J. Goodarzi, and A. T. Johns, "Method to improve digital distance relay impedance measurement when used in series compensated lines protected by a metal oxide varistor,” in Proc. Inst. Elect. Eng., Gen. Transm. Distrib., Jul. 1998, vol. 145, no. 4, pp. 403-508.

[3] D.N. Nkwetta, V. Van Thong, R. Belmans, "Protection of transmission lines using series compensation capacitors in Cameroon-Southern interconnected system, in: proceedings of 3rd IEEE Benelux Young Researchers Symposium in Electrical Power Engineering, 2006, pp5.

[4] T. S. Sidhu and M. Khederzadeh, "Series compensated line protection enhancement by modified pilot relaying scheme,” IEEE Trans. Power Del., vol. 21, no. 3, pp. 1191-1198, Jul. 2006.

[5] P. Jena and A. K. Pradhan, "A positive-sequence directional relaying algorithm for series compensated line,” IEEE Trans. Power Del., vol.25, no. 4, pp. 2288-2297, Oct. 2010.

[6] U.B. Parikh, B. Das, and R.P. Maheshwari, "Combined wavelet-SVM technique for fault zone detection in a series compensated transmission line," IEEE Trans. Power Del., vol. 23, no. 4, pp.1789-1794, Oct. 2008. 
[7] A.I. Megahed, A.M. Moussa, and A.E. Bayoumy, "Usage of wavelet transform in the protection of series-compensated transmission line," IEEE Trans. Power Del., vol. 21, no. 3, pp.1213-1221, Jul. 2006.

[8] Xu Z Y, Su Z P, Zhang J H, et al. "An Interphase Distance Relaying Algorithm for Series-Compensated Transmission Lines”. IEEE Trans. Power Del., vol. 29, no. 2, pp. 834-841, Apr. 2014. 